\title{
A wide-band monolithic differential power amplifier
}

\author{
Ting Tian ${ }^{1}$, Xiaodong Zhang ${ }^{2 a)}$, Huai $\mathrm{Gao}^{3}$, and Shengli Lu ${ }^{1}$ \\ ${ }^{1}$ National ASIC System Engineering Center, Southeast University, Nanjing \\ ${ }^{2}$ School of Electronic \& Information Engineering, Suzhou University of Science \\ and Technology, Suzhou \\ ${ }^{3}$ Suzhou Innotion Tech. Co., Ltd., Suzhou \\ a)zhangxd_usts@126.com
}

Abstract: A differential PA (power amplifier) with a novel structure is introduced in this paper. This PA is constructed with single ended input and balanced output. It is fabricated in $2 \mu \mathrm{m} \mathrm{InGaP/GaAs} \mathrm{HBT} \mathrm{technology.} \mathrm{The}$ small-signal bandwidth of this PA covers from $1.5 \mathrm{GHz}$ to $2.7 \mathrm{GHz} .34 \mathrm{dBm}$ $\mathrm{P}_{-1 \mathrm{~dB}}(1 \mathrm{~dB}$ gain-compression power) and 45\% PAE (power added efficiency) is achieved at $2.1 \mathrm{GHz}$. Moreover, the quiescent power dissipation is $400 \mathrm{~mW}$ with $12 \mathrm{~V}$ supply.

Keywords: differential, push-pull, power amplifier, InGaP/GaAs HBT

Classification: Electron devices, circuits and modules

\section{References}

[1] M. H. Sagor and P. Callaghan: "Benefits of active transmit balanced antenna fed by differential power amplifier," Loughborough Antennas and Propagation Conference (2014) 732 (DOI: 10.1109/LAPC.2014.6996499).

[2] M. P. van der Heijden, et al.: "A $2 \mathrm{GHz}$ high-gain differential InGaP HBT driver amplifier matched for high IP3," IEEE MTT-S International Microwave Symposium Digest 1 (2003) 235 (DOI: 10.1109/MWSYM.2003.1210923).

[3] D. K. Umemoto, et al.: "Integrated npn/pnp GaAs/AlGaAs HBTs grown by selective MBE,” IEEE Electron. Lett. 27 (1991) 1517 (DOI: 10.1049/ el:19910953).

[4] K. W. Kobayashi, et al.: "Integrated complementary HBT microwave push-pull and Darlington amplifiers with p-n-p active loads," IEEE J. Solid-State Circuits 28 (1993) 1011 (DOI: 10.1109/4.237515).

[5] H. Q. Tserng, et al.: "A 0.5-W complementary AlGaAs-GaAs HBT push-pull amplifier at $10 \mathrm{GHz}$," IEEE Microw. Guided Wave Lett. 3 (1993) 45 (DOI: 10. 1109/75.196037).

[6] D. Sawdai and D. Pavlidis: "Push-pull circuits using n-p-n and p-n-p InP-based HBT's for power amplification," IEEE Trans. Microw. Theory Techn. 47 (1999) 1439 (DOI: 10.1109/22.780392).

[7] R. G. Meyer and W. D. Mack: “A wide-band class AB monolithc power amplifier,” IEEE J. Solid-State Circuits 24 (1989) 7 (DOI: 10.1109/4.16295).

[8] M. J. Matilainen, et al.: "An integrated 900-MHz push-pull power amplifier for mobile applications," IEEE MTT-S International Microwave Symposium Digest 2 (2000) 861 (DOI: 10.1109/MWSYM.2000.863316).

[9] H. Gao, et al:: "A new traveling wave matching structure for enhancing the bandwidth of MMIC broadband amplifiers," IEEE Microw. Wireless Compon. Lett. 15 (2005) 508 (DOI: 10.1109/LMWC.2005.852783). 
[10] S. Hu, et al.: "A novel DC-12 GHz variable gain amplifier in $\mathrm{InGaP} / \mathrm{GaAs}$ HBT technology,” IEICE Electron. Express 10 (2013) 20130375 (DOI: 10. 1587/elex.10.20130375).

\section{Introduction}

With continuing growth of personal communications, the number of portable devices with wireless connectivity has increased rapidly. Currently, in the portable devices, a single ended PA feeding an unbalanced antenna is a preferred scheme for the circuit designers. However, this unbalanced design generates a current flow on the ground plane. External objects, such as a human's hand, easily impact the current, the radiation performance of the system is degraded. To solve this problem, an obviously substituted method is using the differential PA and the balanced antenna [1]. It's well known that differential circuits offer an improved linearity and enhanced CMRR (common-mode rejection ratio) [2].

The push-pull amplifier is a basic building block for differential PA. But the research on push-pull amplifier with a complementary P-n-p/N-p-n InGaP/GaAs HBT pair appears being ignored due to the complexity in fabrication $[3,4,5,6]$. A high-efficiency class-AB pseudo-push-pull amplifier designed with N-p-n HBTs alone can be realized by using a 180-degree phase splitters (or baluns) [7, 8]. Due to the incomplete symmetry of the two branches, the difference in output signal between the positive and negative half-cycle of the waveform is relatively obvious. The use of a differential topology can improve the above phenomenon.

In this paper, a novel monolithic differential PA with single ended input and balanced output is proposed. The PA is fabricated in $2 \mu \mathrm{m} \mathrm{InGaP/GaAs} \mathrm{HBT}$ technology, which achieves a bandwidth from $1.5 \mathrm{GHz}$ to $2.1 \mathrm{GHz}$, a $\mathrm{P}_{-1 \mathrm{~dB}}$ of $34 \mathrm{dBm}$ and PAE of $45 \%$ at $2.1 \mathrm{GHz}$. The output stage of the PA is biased on class $\mathrm{AB}$, so the PA has a low quiescent current and dissipates only $400 \mathrm{~mW}$ quiescent power with $12 \mathrm{~V}$ supply.

\section{Circuit design}

\subsection{Circuit topology}

The circuit architecture of the proposed differential PA is shown in Fig. 1. This differential PA consists of an input stage and two push-pull amplifiers. A1, A2, Q1 and Q2 compose a push-pull amplifier. Another exactly same push-pull circuit includes A3, A4, Q3 and Q4. A0 is the input stage which drives the next two pushpull amplifiers with opposite phase.

In the positive half cycle of the input signal, the transistor Q1 and Q4 are turned on. Simultaneously, the transistor Q2 and Q3 are turned off. Thus, the current flows from Q1 to Q4. On the contrary, the current flows from Q3 to Q2 in the negative half cycle of the input signal.

\subsection{Push-pull amplifier}

Fig. 2 presents the schematic of push-pull amplifier. This is a wide-band Class AB amplifier. Power device Q1 and Q2 are the last output stage. Q5 is a common 


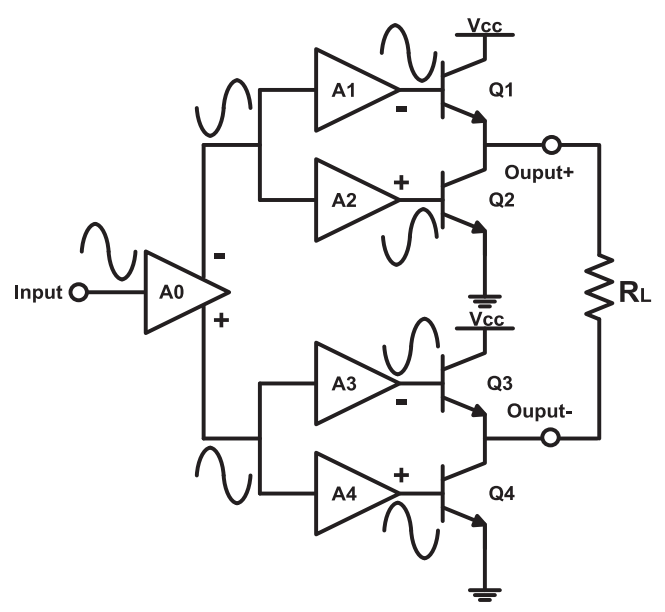

Fig. 1. Simplified circuit topology structure

emitter amplifier, as the inverse amplifier A1 in Fig. 1, which drives Q1. Q6 is an emitter follower, as the amplifier A2 in Fig. 1, which drives Q2. D1 and C1 are used to shift the DC input level of Q5. Thus, Q5 and Q6 can share a bias circuit composed by Q7, Q8, Q9 and D2. To achieve high power efficiency, the circuit is designed for a low idling current of $15 \mathrm{~mA}$ in Q1 and Q2, by adjusting the resistor $\mathrm{R} 2, \mathrm{R} 3$ and R4.

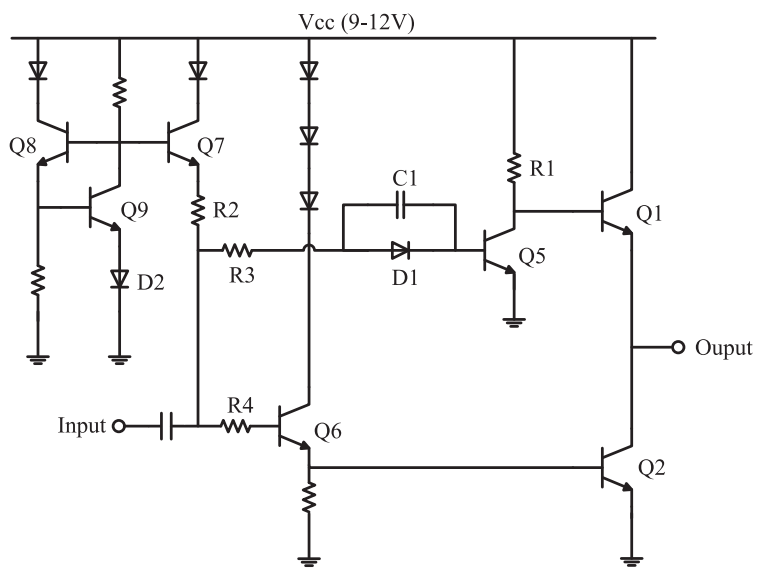

Fig. 2. Schematic of push-pull amplifier

Obviously, in the push-pull amplifier, the two half-cycles are magnified by different circuits. So it is impossible to make the voltage gain of two half-cycles equal in the whole temperature range and frequency range. However, the proposed differential PA in Fig. 1 allows preventing from the difference between the positive and negative half-cycle of the output waveform. Fig. 3 shows the simulated results of the output waveform of the push-pull structure and differential structure. With a $7 \mathrm{dBm}$ input power at $2.1 \mathrm{GHz}$, the difference in output signal between the positive and negative half-cycle of the waveform of the push-pull structure is $1.357 \mathrm{~V}$, while $0.919 \mathrm{~V}$ of the differential structure. 


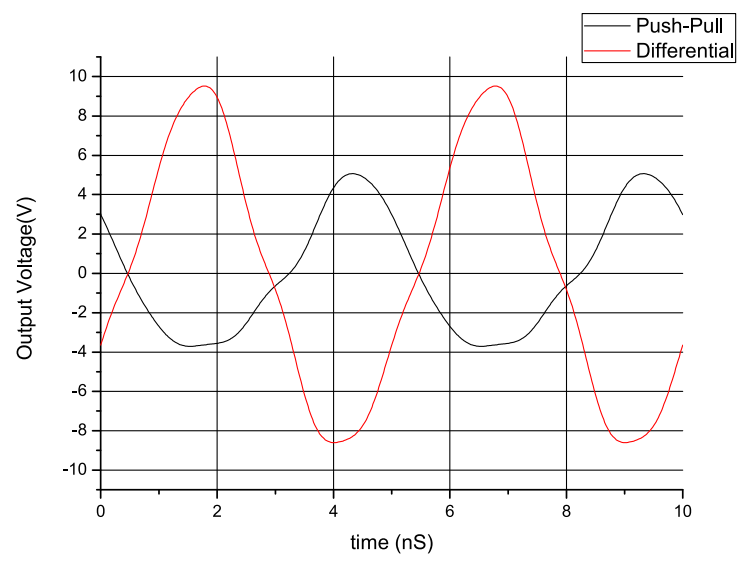

Fig. 3. Output signal of the push-pull and differential structure

\subsection{Input stage design}

The PA's first stage is shown in Fig. 4. In this circuit, R3, R4 and D2 offer the bias voltage for the transistor Q0. The signals $\mathrm{v}-$ and $\mathrm{v}+$ output from the collector and emitter of transistor Q0 separately. If the base current of Q0 can be ignored, the signals $\mathrm{v}-$ and $\mathrm{v}+$ will swing with same amplitude and opposite phase, when the resistor R5 equals to R6. However, in order to acquire the better performance in high frequency, the base-emitter and base-collector junction capacitance in Q0 have to be taken into account.

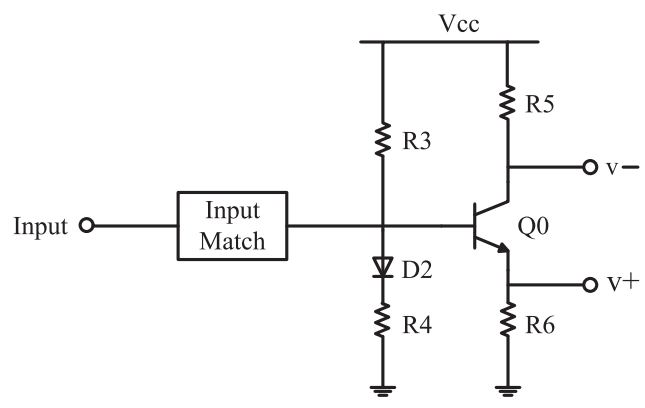

Fig. 4. Schematic of input stage

The elements in Fig. 5 are as follows.

1) gbe and Cbe are base-emitter conductance and capacitance of Q0.

2) $\mathrm{Cbc}$ is base-collector capacitance of Q0.

3) $\mathrm{rbb}$ and gm are intrinsic base resistance and transconductance of Q0.

4) R5 and R6 are the impedance connected with collector and emitter.

5) rpp and Cpp are the input resistance and input capacitance of the following push-pull amplifier.

The parameters of the input stage equivalent circuit are shown in Table I.

Write the following equations using Kirchoff's current laws (KCL):

$$
\left\{\begin{array}{l}
-v_{e} Y_{e}+\left(v_{b^{\prime}}-v_{e}\right)\left(g_{m}+g_{b e}+s c_{b e}\right)=0 \\
\left(v_{c}-v_{b^{\prime}}\right) s c_{b c}+v_{c} Y_{c}+\left(v_{b^{\prime}}-v_{e}\right) g_{m}=0
\end{array}\right.
$$

Based on the KCL above, in order to satisfy the requirement of ve $=-\mathrm{vc}$, formula (2) can be derived. According to formula (2) and the parameters in Table I, 


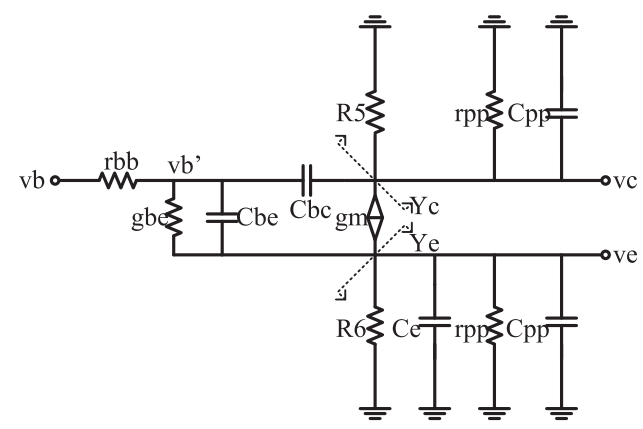

Fig. 5. Equivalent circuit of input stage

Table I. Parameters of the equivalent circuit

\begin{tabular}{|c|c|c|c|c|c|c|c|}
\hline gbe & gm & Cbe & Cbc & rpp & Cpp & R5 & R6 \\
\hline $0.84 \mathrm{mS}$ & $66 \mathrm{mS}$ & $0.42 \mathrm{pf}$ & $0.04 \mathrm{pf}$ & $83 \mathrm{ohm}$ & $0.21 \mathrm{pf}$ & $80 \mathrm{ohm}$ & $80 \mathrm{ohm}$ \\
\hline
\end{tabular}

a small capacitance $\mathrm{Ce}(0.26 \mathrm{pf})$ shunted beside the resistance $\mathrm{R} 6$ can lead to better symmetry between ve and vc

$$
Y_{e}-Y_{c}=\frac{Y_{c}\left(g_{b e}+s c_{b e}+s c_{b c}\right)+2 s c_{b c}\left(g_{m}+g_{b e}+s c_{b e}\right)}{g_{m}-s c_{b c}}
$$

\subsection{Input matching}

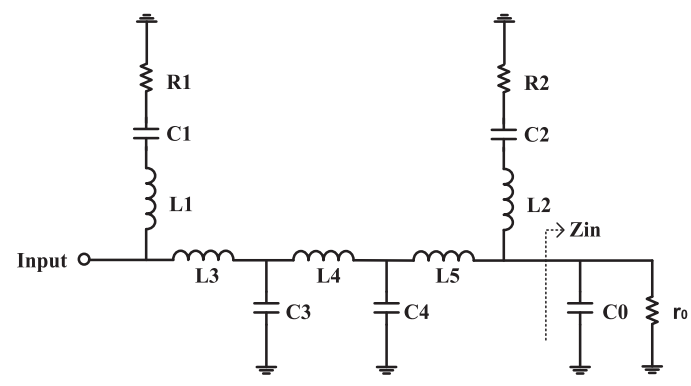

Fig. 6. Equivalent circuit of input matching

A traveling wave matching structure is used to enhance the bandwidth of amplifier $[9,10]$. The input matching equivalent circuit is shown in Fig. 6. The input impedance of input stage can be simplified as parallel $\mathrm{C} 0$ and $\mathrm{r}_{0}$. For broadband input impedance matching, a tapered artificial transmission line with three sections is introduced before the input stage. The equivalent capacitance $\mathrm{C} 0$ is incorporated with the on chip lumped elements, L3, L4, L5, C3 and C4, to form the lossless artificial transmission line. The characteristic impedance of the line sections, $r_{1}, r_{2}$ and $r_{3}$, are determined by the following.

$$
r_{3}=r_{0}=\sqrt{\frac{L_{5}}{c_{0}}}, \quad r_{1}=r_{s}=\sqrt{\frac{L_{3}}{c_{3}}}, \quad r_{2}=\sqrt{r_{1} r_{3}}=\sqrt{\frac{L_{4}}{c_{4}}}
$$

Where, $r_{\mathrm{s}}$ is the input source impedance.

The parameters of the input matching circuit are shown in Table II. 
Table II. Parameters of the input matching circuit

\begin{tabular}{|c|c|c|c|c|c|c|c|}
\hline $\mathrm{r}_{\mathrm{s}}$ & $\mathrm{r}_{0}$ & $\mathrm{C} 0$ & $\mathrm{C} 3$ & $\mathrm{C} 4$ & $\mathrm{~L} 3$ & L4 & L5 \\
\hline $50 \mathrm{ohm}$ & $120 \mathrm{ohm}$ & $0.3 \mathrm{pf}$ & $0.3 \mathrm{pf}$ & $0.3 \mathrm{pf}$ & $0.75 \mathrm{nH}$ & $1.8 \mathrm{nH}$ & $4.32 \mathrm{nH}$ \\
\hline
\end{tabular}

Furthermore, two RLC compensation network are connected to the artificial transmission line, which form an M-Type filter to improve the matching performance for high and low frequency band. The high frequency compensation network is composed of L1, C1, and R1. The low frequency compensation network is composed of L2, C2, and R2.

\subsection{Output matching}

This differential PA is with balanced output. The measurement system is generally single-ended system. Transmission-line transformers are well known for their wide bandwidth. So, in the output matching, a 1:4 transformer manufactured by RFMD, RFXF8553 is employed as a double-end and single-end conversion circuit.

\section{Measurement result}

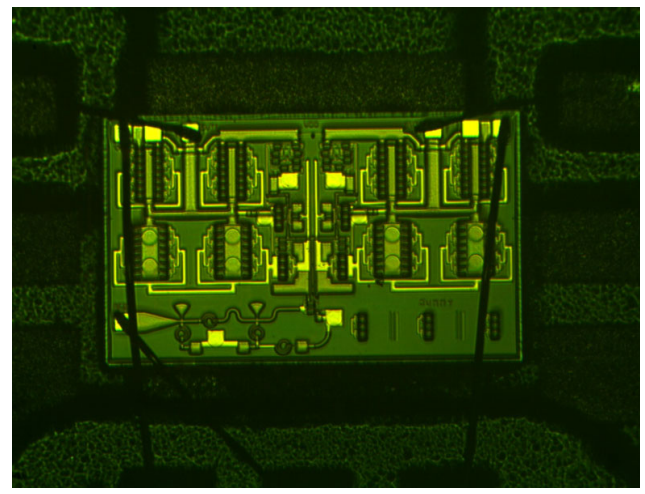

Fig. 7. Die photo of the proposed differential PA

The proposed differential PA was implemented in a commercial $\mathrm{InGaP} / \mathrm{GaAs}$ HBT technology. The HBT emitter area is $2 \times 20 \mu \mathrm{m}^{2}$. The chip size is $0.9 \times 1.5 \mathrm{~mm}^{2}$. The die photo is shown in Fig. 7.

The measurement broadband small signal gain and return loss are plotted in Fig. 8 (a). The small signal gain is $27 \mathrm{~dB}$ at $2.1 \mathrm{GHz}$ and the input return loss is better than $15 \mathrm{~dB}$ from $1.78 \mathrm{GHz}$ to $2.67 \mathrm{GHz}$. Fig. 8 (b) shows the measurement results of power gain vs. input power at three typical frequencies. Fig. 8(c) shows the measurement result of the $1-\mathrm{dB}$ output power vs. frequency. Fig. $8(\mathrm{~d})$ shows the measurement result of PAE vs. output power. $34 \mathrm{dBm} \mathrm{P}-1 \mathrm{~dB}$ and $45 \%$ PAE is achieved at $2.1 \mathrm{GHz}$. 


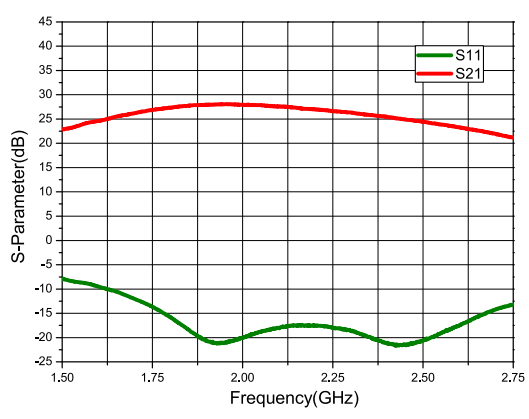

(a)

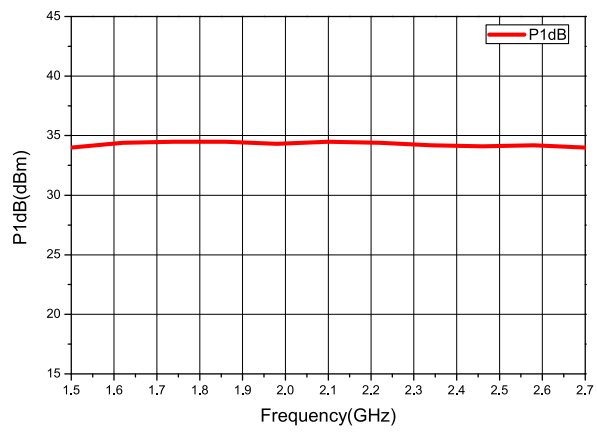

(c)

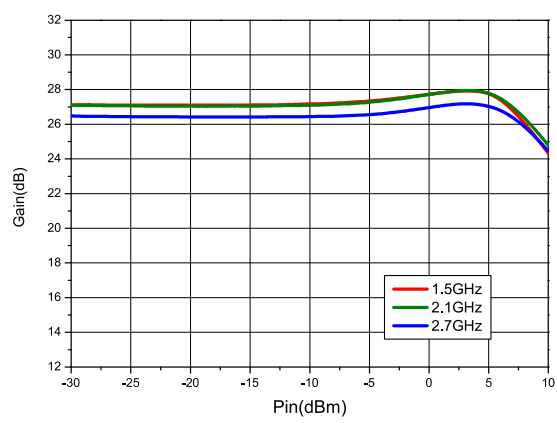

(b)

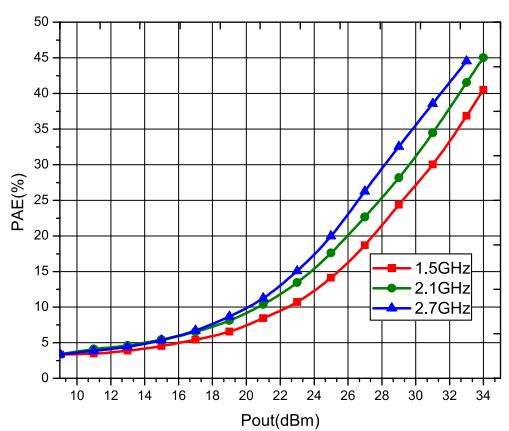

(d)

Fig. 8. (a) Broadband gain \& return loss. (b) Power gain vs. input power. (c) $\mathrm{P}_{-1 \mathrm{~dB}}$ vs. frequency. (d) PAE vs. output power.

\section{Conclusion}

In this letter, a novel differential PA fabricated in $2 \mu \mathrm{m}$ InGaP/GaAs HBT technology is proposed. It is target for a wide band frequency application from 1.5 GHz to $2.7 \mathrm{GHz}$. The novel differential PA consists of an input stage and two push-pull amplifiers. Only N-type active devices are used in circuit design. $34 \mathrm{dBm}$ $\mathrm{P}_{-1 \mathrm{~dB}}$ and $45 \% \mathrm{PAE}$ are achieved at $2.1 \mathrm{GHz}$. 